\title{
STABLE CLIFF CLIMBING FOR HEXAPOD ROBOT WITH ARTICULATED BODY
}

\author{
A. V. PANCHENKO \\ Faculty of Mechanics and Mathematics, Lomonosov Moscow State University, \\ Moscow, 119991, Russia \\ E-mail: PanchenkoAV@vap.ru \\ V. E. PAVLOVSKY \\ Faculty of Mechanics and Mathematics, Lomonosov Moscow State University, \\ Moscow, 119991, Russia \\ E-mail:vlpavl@mail.ru
}

The paper describes kinematic control for hexapod robot with three-segment articulated body. Forward and inverse kinematics for articulated body described. Static stability studied in case of climbing the so-called cliff obstacle. Conditions for static stability during climbing sequence provided.

Keywords: Multi-legged robot; obstacle climbing; articulated body; simulation.

\section{Introduction}

Study of walking machines is a rather old question. Starting from Ancient China mechanical designs ${ }^{1}$ and ending with state of the art reinforcement learning ${ }^{2}$ and model predictive control approaches. ${ }^{3}$ Multi-legged walking robots are complicated systems in terms of control and planning due to significant number of degrees of freedom (d.o.f.) and actuators, complexity of the environment and etc. Nowadays the most complex and robust walking machines are made by Boston Dynamics company. ${ }^{4}$ Its robots are capable of working in human environment and outdoors. The basis of their approach is model predictive control and non-linear optimization.

The main subject of article is to find simple approaches for building control algorithms for multi-legged walking robots with articulated body. It is clear that robots with flexible body can overcome obstacles that are more complex.

For that purpose, a hexapod robot with three-segment body was studied 
in task of overcoming a cliff obstacle using Coulomb forces with coefficient of static friction less than 1.

Static stability equations were resolved for robot in different configurations with additional assumptions of symmetry. Motion sequence for climbing on a cliff with static stability preservation was build and verified in computer simulation.

The designed approach for static stability study can be further used for other types of obstacles, such as narrow passage, sharp ditch, windrow and so on. Besides of that, the approach can be applied for different models of robot's body without any modifications.

\section{Robot Kinematics}

Let us consider robot depicted on figure Fig. 1. It has six so-called insectomorphic legs, i.e. insect-like leg kinematics. Each leg has three degrees of freedom. Body consists of three rigid segments connected with hinges to each other. Body that consists of several segments connected to each other with controllable joints and can change its geometry is called articulated.

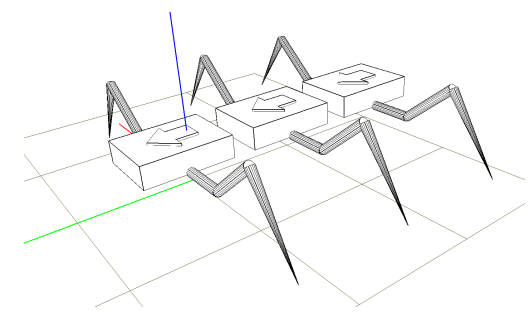

Fig. 1. Hexapod robot with articulated body.

The total number of degrees of freedom (d.o.f.) for specified robot is 26 :

- 3 d.o.f. for each leg, i.e. 18 d.o.f. for all legs;

- 2 d.o.f. for body segments;

- 6 d.o.f. for the whole system as one single body.

Overall it's 26 d.o.f. and 20 of them can be controlled with actuators installed in corresponding rotational joints. Leg kinematics is well-known and was already studied in all details. 


\section{Cliff Obstacle}

Cliff obstacle consists of three planes two of which are horizontal and one is vertical as depicted on Fig. 2.

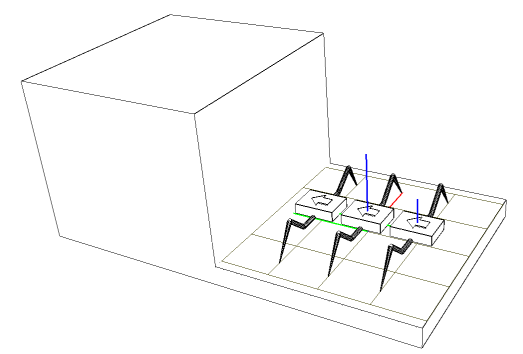

Fig. 2. Cliff obstacle.

The distance between two horizontal planes is equal to $H$. Robot starts from the lower horizontal plane and his goal is to climb up the higher horizontal plane using only the Coulomb friction. To overcome cliff obstacle robot moves using so-called gallop gait when a pair of symmetrical legs from left and right sides of the robot are in transition state and the others are in support state, i.e. in every moment of time there are four legs in contact with obstacle. Body kinematics will be considered in next section.

\section{Body Kinematics}

Robot's body consists of three equal rigid segments connected to each other with rotational joints with axes aligned in lateral direction of the body. Each segment has a pair of legs connected. Additional joints with angles $\delta_{1}$ and $\delta_{2}$ in the robots body allow to bend body and follow the surface shape, shift legs mounting points towards to supporting surface. To find body joint angles the following procedure is defined.

Initial and goal poses for middle segment are connected with a cubic spline curve, which represents target trajectory for middle segment. If segments and their trajectory are known, then the task is solved through simple linear approximation as depicted on Fig. 3.

To keep the contact points on the goal trajectory all joints should act in a coordinated way. At every moment of time all joint coordinates must be 


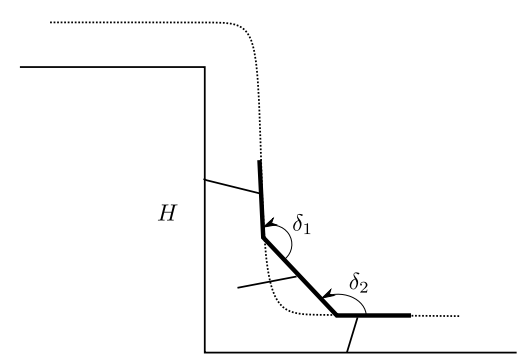

Fig. 3. Body inverse kinematics through simple linear approximation.

updated to keep the end effectors at the goal position. Additional mobility inside the robots body should be taken into account because all legs are connected to the different segments. Target point $\bar{R}_{i}$ for $i$-th leg is given in global reference frame. To obtain leg joint angles the inverse kinematic equations are used, point $\bar{R}_{i}$ must be translated into legs reference frame. To manage all relative coordinate transformations of shifts and rotations between body segments, legs and joints, homogeneous coordinates are used. Calculation of all coordinate transformation for each leg at every moment of time can be easily done automatically through well-known kinematics of robot. The main differences between articulated body and single segment body are:

- Higher ability to overcome obstacles - segments follow the surface;

- Articulated body is able to shift mounting points of its legs - service region is not a constant, i.e. in some conditions robot can reach contact surface and put legs on it;

- Center of gravity is shifted in a wider range with all else parameters being equal - critical parameter in static stability preservation in extreme conditions.

\section{Cliff Climbing Stability}

The system is stable when sums of all external forces and all momentums are equal to zero.

$$
\left\{\begin{array}{c}
\sum_{i=1}^{N} \bar{R}_{i}+\bar{P}=0 \\
\sum_{i=1}^{N}\left[\bar{r}_{i} \times \bar{R}_{i}\right]+\left[\bar{r}_{c} \times \bar{R}_{c}\right]=0
\end{array}\right.
$$


The following configurations of supporting legs displacement should be studied for static stability:

- All legs on some horizontal plane. This case is already well studied;

- Front legs lean against the vertical plane and rear legs stand on the lower horizontal plane. Let us refer to this configuration as Number One.

- Front legs are placed at the upper horizontal plane, while the rear legs stand on the vertical plane. Let us refer to this configuration as Number Two.

- All legs stand on the upper horizontal plane - this case is similar to the initial one.

Considering the robot as a slow moving system at every moment of time let us find conditions for static stability. Reference frame Oxyz is defined as depicted on Fig. 4.

\subsection{First Configuration}

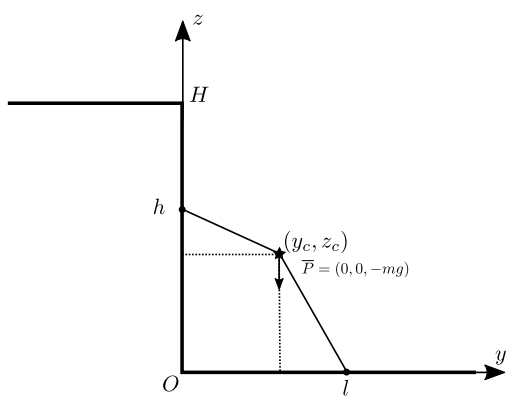

Fig. 4. First static configuration.

Contact points of the legs for first configuration are as follows:

$$
\bar{r}_{1}=(d, 0, h), \bar{r}_{2}=(-d, 0, h), \bar{r}_{3}=(d, l, 0), \bar{r}_{4}=(-d, l, 0) .
$$

There is a reaction $\bar{R}_{i}$ acting on the robots legs at each contact point:

$$
\bar{R}_{i}=N_{i} \cdot \bar{n}_{i}+F_{\tau}^{i} \cdot \bar{\tau}_{i}+F_{\nu}^{i} \cdot \bar{\nu}_{i}
$$

The $\bar{\tau}_{i}$ and $\overline{n u}_{i}$ vectors have the following coordinates: 


$$
\begin{aligned}
& \bar{n}_{1}=(0,1,0), \bar{\tau}_{1}=(0,0,1), \bar{\nu}_{1}=(1,0,0), \\
& \bar{n}_{2}=(0,1,0), \bar{\tau}_{2}=(0,0,1), \bar{\nu}_{2}=(1,0,0), \\
& \bar{n}_{3}=(0,0,1), \bar{\tau}_{3}=(0,1,0), \bar{\nu}_{3}=(1,0,0), \\
& \bar{n}_{4}=(0,0,1), \bar{\tau}_{4}=(0,1,0), \bar{\nu}_{4}=(1,0,0) .
\end{aligned}
$$

The center of gravity has coordinates:

$$
\bar{r}_{c}=\left(0, y_{c}, z_{c}\right)
$$

The gravity force $\bar{P}$ acts on the center of gravity of the robot:

$$
\bar{P}=(0,0,-P)
$$

The equations of static stability for first configuration are as follows:

$$
\left\{\begin{aligned}
F_{\nu}^{1}+F_{\nu}^{2}+F_{\nu}^{3}+F_{\nu}^{4} & =0 \\
N_{1}+N_{2}+F_{\tau}^{3}+F_{\tau}^{4} & =0 \\
F_{\tau}^{1}+F_{\tau}^{2}+N_{3}+N_{4} & =P \\
N_{1} h+N_{2} h+P y_{c} & =l\left(N_{3}+N_{4}\right) \\
d\left(F_{\tau}^{1}+N_{3}\right) & =F_{\tau}^{2} d+F_{\nu}^{1} h+F_{\nu}^{2} h+N_{4} d \\
d\left(F_{\tau}^{3}+N_{1}\right) & =F_{\tau}^{4} d+N_{2} d+F_{\nu}^{3} l+F_{\nu}^{4} l
\end{aligned}\right.
$$

The total number of equation is six. The number of unknown variables is twelve. Let us assume that the friction forces are modelled with Coulomb mathematical model:

$F_{j}^{i}=k_{j}^{i} \cdot N_{i}$, where $k_{j}^{i}$ is coefficient of friction for $i$-th leg in $j$-th direction.

After substitution of the Coulomb friction model, the Eq. (7) will transform into the following system:

$$
\left\{\begin{aligned}
& N_{1} k_{\nu}^{1}+N_{2} k_{\nu}^{2}+N_{3} k_{\nu}^{3}+N_{4} k_{\nu}^{4}=0 \\
& N_{1}+N_{2}+N_{3} k_{\tau}^{3}+N_{4} k_{\tau}^{4}=0 \\
& N_{3}+N_{4}+N_{1} k_{\tau}^{1}+N_{2} k_{\tau}^{2}=P \\
& N_{1} h+N_{2} h+P y_{c}=l\left(N_{3}+N_{4}\right) \\
& d\left(N_{3}+N_{1} k_{\tau}^{1}\right)=d N_{4}+d N_{2} k_{\tau}^{2}+h N_{1} k_{\nu}^{1}+h N_{2} k_{\nu}^{2}, \\
& d\left(N_{1}+N_{3} k_{\tau}^{3}\right)=d N_{2}+d N_{4} k_{\tau}^{4}+l N_{3} k_{\nu}^{3}+l N_{4} k_{\nu}^{4}
\end{aligned}\right.
$$

The number of unknowns variables remains the same, and besides $N_{i}>0$. Let us introduce additional assumptions that the left and the right side of the robot are loaded equally and coefficients of friction are the same between left and right legs: 


$$
\left\{\begin{array}{c}
k_{\nu}^{1}=-k_{\nu}^{2}=k_{\nu}, \\
k_{\nu}^{3}=-k_{\nu}^{4}=k_{\nu}, \\
k_{\tau}^{1}=k_{\tau}^{2}=k_{\tau}^{u}, \\
k_{\tau}^{3}=k_{\tau}^{4}=k_{\tau}^{d}, \\
N_{1}=N_{3}=N_{u}, \\
N_{2}=N_{4}=N_{d} .
\end{array}\right.
$$

The system of three equations and four variables obtained:

$$
\left\{\begin{aligned}
N_{u}+N_{d} k_{\tau}^{d} & =0, \\
2 N_{d}+2 N_{u} k_{\tau}^{u} & =P, \\
2 h N_{u}+P y_{c} & =2 l N_{d} .
\end{aligned}\right.
$$

Number of unknowns is still greater than number of equations. One more assumption must be introduced:

$$
k_{\tau}^{u}=k_{\tau}^{d}=k_{\tau}>0
$$

Finally, the system of three equations and three unknowns is obtained:

$$
\left\{\begin{aligned}
N_{u} & =N_{d} k_{\tau} \\
2 N_{d}+2 N_{u} k_{\tau} & =P \\
2 h N_{u}+P y_{c} & =2 l N_{d}
\end{aligned}\right.
$$

Let us find unknown reactions $N_{u}, N_{d}$ and $k_{\tau}$. From first and second equations of Eq. (12) follows:

$$
\begin{aligned}
N^{u} & =N^{d} k_{\tau}, \\
N^{d} & =\frac{P}{2\left(1+k_{\tau}^{2}\right)} .
\end{aligned}
$$

After substituting Eq. (13) to the third equation of Eq. (12) we have quadratic equation relative to $k_{\tau}$ :

$$
y_{c} k_{\tau}^{2}+h k_{\tau}+\left(y_{c}-l\right)=0
$$

There are two solutions for quadratic Eq. (14), but only one satisfies condition $0<k_{\tau}<1$ :

$$
0<k_{\tau}=-\frac{h-\sqrt{h^{2}-4 y_{c}^{2}+4 l y_{c}}}{2 y_{c}}<1
$$




\subsubsection{Dimensionless parameters}

It is easy to see that the Eq. (15) for $k_{\tau}$ depends on $l, h$ and $y_{c}$ parameters that are measured in meters they all have the same physical dimension. Let us use this circumstance and define the following dimensionless parameters:

$$
p_{1}:=\frac{h}{y_{c}}, p_{2}:=\frac{l}{y_{c}} \text {, where } y_{c} \neq 0 .
$$

After solving Eq. (15) with substituted Eq. (16), the solution of Eq. (15) is depicted on Fig. 5.

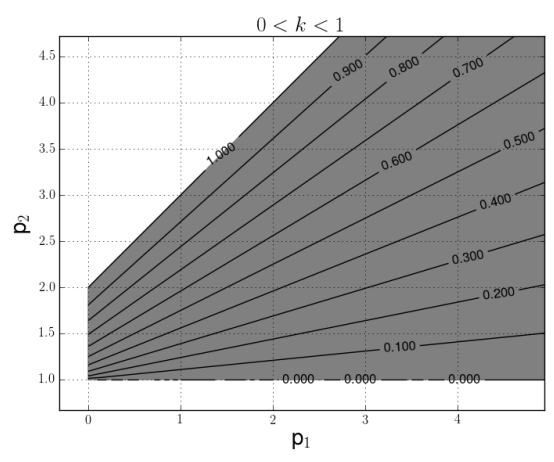

Fig. 5. Solution of Eq. (15) with labeled contour lines for $k_{\tau}$ as a function of $\left(p_{1}, p_{2}\right)$.

From Fig. 5 it becomes clear that if there is lack of friction in contact points the robot should:

- move its center of gravity closer to the rear legs;

- choose contact points higher on the vertical plane for front legs;

- choose contact points closer to vertical plane for rear legs.

\subsection{Second Configuration}

Leg contact points for the second configuration are:

$$
\begin{aligned}
& \bar{r}_{1}=(d, l, H), \bar{r}_{2}=(-d, l, H) \\
& \bar{r}_{3}=(d, 0, h), \bar{r}_{4}=(-d, 0, h)
\end{aligned} \text {, where } l<0, H>h, y_{c}>l .
$$

Similarly, for the second configuration we get the following system of three equations: 


$$
\left\{\begin{aligned}
N_{u}-N_{d} k_{\tau} & =0, \\
2 N_{u}+2 N_{d} k_{\tau} & =P, \\
2 N_{d} h+P y_{c} & =2 N_{u}\left(l+H k_{\tau}\right)
\end{aligned}\right.
$$

There are two possible solutions for $k_{\tau}$, but only one meets requirement $0<k_{\tau}<1$ :

$$
0<k_{\tau}=\frac{(H-h)-\sqrt{(H-h)^{2}-4 y_{c}^{2}+4 l y_{c}}}{2 y_{c}}<1
$$

\subsubsection{Dimensionless parameters}

Let us find solution of inequality $0<k_{\tau}<1$ using the following dimensionless parameters:

$$
p_{1}:=\frac{(H-h)}{y_{c}}, p_{2}:=\frac{l}{y_{c}} \text {, where } y_{c} \neq 0 .
$$

The solution of Eq. (19) is depicted on Fig. 6.
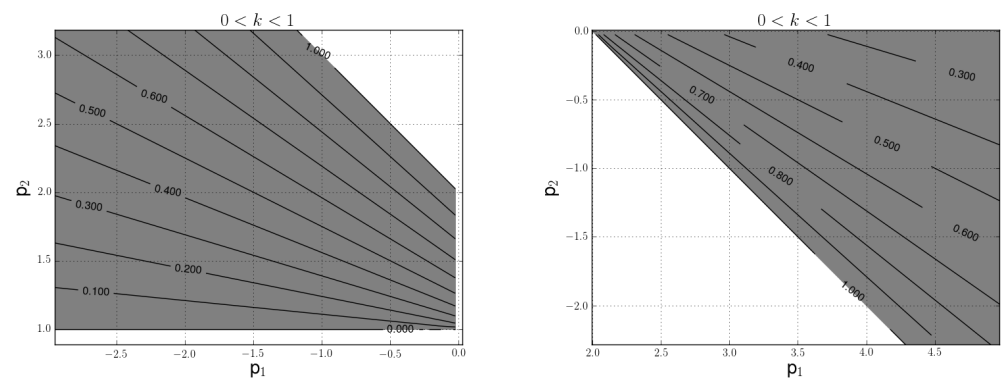

Fig. 6. Solution of Eq. (19). Left $-y_{c}<0$, right $-y_{c}>0$.

From Fig. 6 it can be shown that for case when $y_{c}>0$ to reduce the value of $k_{\tau}$ robot should:

- keep its center of gravity far from front legs;

- keep rear legs as low as possible;

- keep front legs closer to cliff edge. 
From Fig. 6 it can be shown that for case when $y_{c}<0$ to reduce the value of $k_{\tau}$ robot should:

- keep its center of gravity closer to front legs;

- keep rear legs as low as possible;

- keep front legs closer to cliff edge.

In Eq. (20) we have considered substitution in assumption that $y_{c} \neq 0$. Let us see what happens when center of gravity is right above the cliffs edge in second configuration.

\subsubsection{Second configuration. $y_{c}$ equals to zero}

If the $y_{c}=0$ the equations of static stability Eq. (18) will transform into the following system:

$$
\left\{\begin{array}{l}
N_{d}-N_{u} k_{\tau}=0 \\
2 N_{u}+2 N_{d} k_{\tau}=P \\
N_{d} h-N_{u} l=H N_{u} k_{\tau}
\end{array}\right.
$$

There is only one solution for $k_{\tau}, N_{u}$ and $N_{d}$ for Eq. (21):

$$
\left\{\begin{aligned}
k_{\tau} & =-\frac{l}{H-h}, \\
N_{u} & =\frac{P(H-h)^{2}}{2\left((H-h)^{2}+l^{2}\right)} \\
N_{d} & =\frac{-P l(H-h)}{2\left((H-h)^{2}+l^{2}\right)}
\end{aligned}\right.
$$

Due to Eq. (17) the Eq. (22) for $k_{\tau}$ is always greater than zero. From the other side, the requirement $k_{\tau}<1$ is equivalent to the following inequality:

$$
0<-l<(H-h) \text {, where } l<0
$$

Equation (23) means that to provide stable configuration in case when $y_{c}=0$ the contact points should be chosen in a way, that the front legs should be closer to cliff edge than the rear legs.

\section{Hexapod simulation for cliff climbing}

To verify the static stability conditions the computer simulation of articulated hexapod was made. The "Universal Mechanism" ${ }^{5}$ software package 
was used to provide dynamical model of the articulated hexapod robot and the cliff obstacle. All robot's legs have so called viscous elastic point contact models with the cliff obstacle.

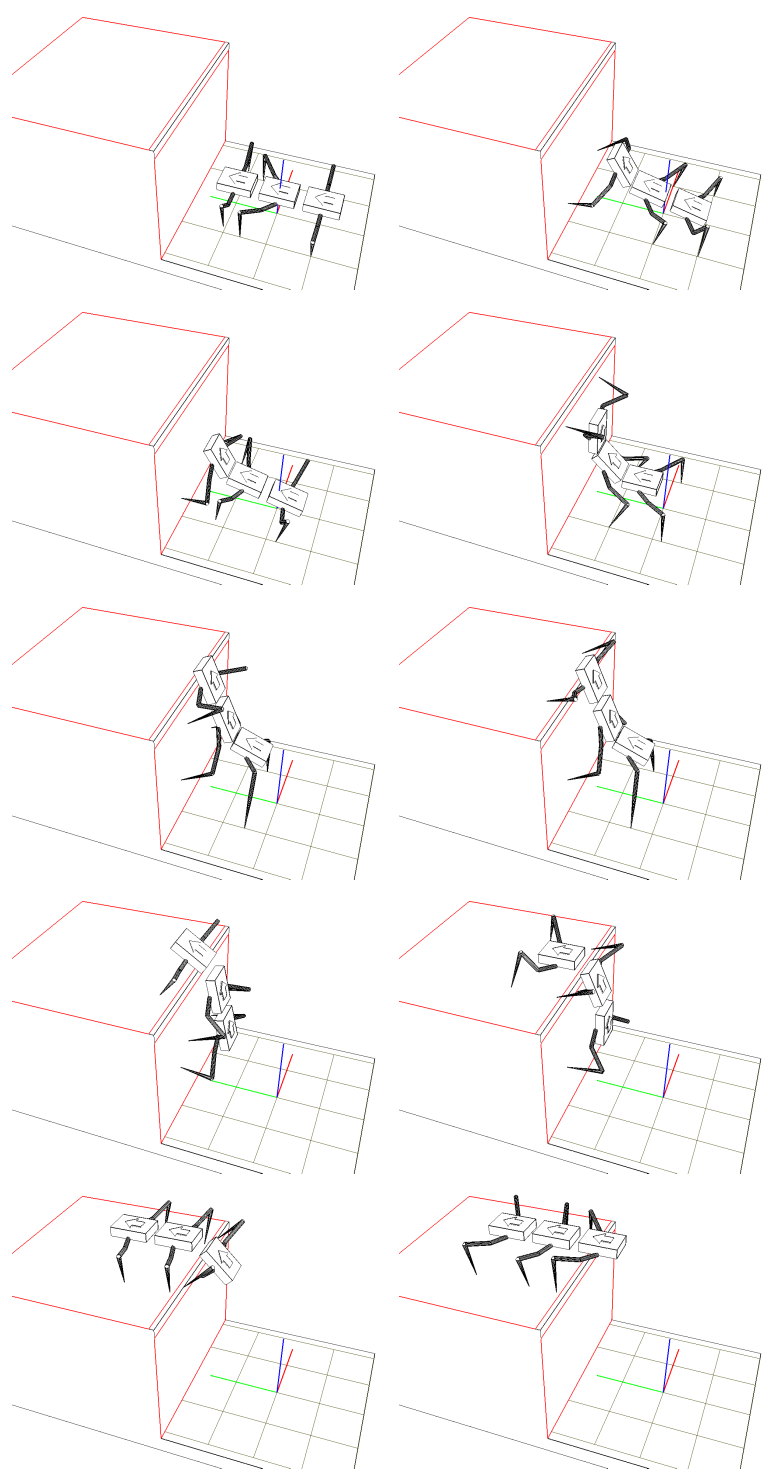

Fig. 7. Climbing motion sequence (left-to-right, top-to-bottom). 
The desired robot motion was manually scripted as list of scheduled commands for leg's positions and body configuration along with position and orientation. Robot's climbing motion sequence during simulation is depicted on Fig. 7. The resulting time of climbing maneuver in quasi static regime of motion takes about 50 seconds to climb up the cliff. The ratio between real and simulation time is approximately equal to 1 on computer with Core-i7 CPU and GTX950M GPU.

\section{Conclusion}

The analysis of robot's configurations in different poses on the cliff proved that stable quasi static motion is possible for all steps, i.e. the robot is capable of climbing the cliff with static stability preservation using only Coulomb friction forces. Articulated three segment body has shown advantages in comparison with similar rigid body in context of extreme obstacle overcoming. On the other hand, increased passability requires more complex motion planning and control. Conditions for statics stability for all main climbing configurations obtained.

\section{Acknowledgements}

The research work is supported by the Russian Foundation for Basic Research. The unique project number is 16-31-00524 mol_a.

\section{References}

1. H.-S. Yan, Journal of the Chinese Society of Mechanical Engineers 26, 133 (2005).

2. X. B. Peng, G. Berseth, K. Yin and M. Van De Panne, ACM Transactions on Graphics (TOG) 36, p. 41 (2017).

3. H. Diedam, D. Dimitrov, P.-B. Wieber, K. Mombaur and M. Diehl, Online walking gait generation with adaptive foot positioning through linear model predictive control, in Intelligent Robots and Systems, 2008. IROS 2008. IEEE/RSJ International Conference on, (Nice, France, 2008).

4. M. Raibert, K. Blankespoor, G. Nelson and R. Playter, IFAC Proceedings Volumes 41, 10822 (2008).

5. Universal mechanism http://www.umlab.ru/en.

6. Y. F. Golubev and V. Koryanov, Journal of Computer and Systems Sciences International 47, 139 (2008).

7. Y. F. Golubev and V. Koryanov, Journal of Computer and Systems Sciences International 48, p. 801 (2009).

8. A. V. Panchenko, I. A. Orlov and V. E. Pavlovsky, Control algorithm for walking robot with mosaic body, in ASSISTIVE ROBOTICS: Proceedings 
of the 18th International Conference on CLAWAR 2015, (Hangzhou, China, 2016).

9. Y. F. Golubev, V. V. Korianov, V. E. Pavlovsky and A. V. Panchenko, Motion control for the 6-legged robot in extreme conditions, in Nature-Inspired Mobile Robotics, (Sydney, Australia, 2013). 